\title{
Suitability of Indoor Positioning System for Smart City loT Applications
}

\author{
Sania Mushtaq \\ Dept. of Telecommunication \\ Engineering, UET, Taxila, \\ 47050, Pakistan
}

\author{
Adeel Akram, PhD \\ Dept. of Telecommunication \\ Engineering, UET, Taxila, \\ 47050, Pakistan
}

\author{
Saba Farooq Abbasi \\ Dept. of Telecommunication \\ Engineering, UET, Taxila, \\ 47050, Pakistan
}

\begin{abstract}
Indoor Positioning is a substantial field of research which requires frequent revisions due to continual developments and various applications in the Smart-City environment. It has found its way into various applications because of the consistent requirement of increased positioning accuracy while maintaining the user privacy. This paper presents technological overview and comparison of indoor location systems for their suitability in Smart City applications. These systems are analyzed for their scalability, range, accuracy, storage requirements and privacy. The paper is fixated on indoor positioning systems that have high accuracy.
\end{abstract}

\section{General Terms}

Smart Cities, Indoor Positioning, Privacy, Scalability, Mobility

\section{Keywords}

Internet of Things (IoT), Indoor Positioning (IP), Location Awareness, Cricket, Active Bat, Active Badge, Ubisense, FIND.

\section{INTRODUCTION}

Through the realization and progression of the Internet of Things (IoT), pervasive computing is evolving into one of the most prominent grounds for research. Development of pervasive system connectivity and network assisted devices played a major role in ubiquitous computing. Smart networking and circumstantial awareness of existing systems with controlled rates and low power solutions are the principal needs for IoT [1].

Smart City is described as a town or a city that escalates the functional ability by integrating data and transmission technologies. In order to lessen global expenses, source depletion and expenditure the enactment and excellence of metropolitan facilities (conveyance, energy etc.) is enriched [2]. Smart City comprises smart services, smart industry, smart homes, smart technology and smart management as the crucial modules of inner-city growth. To form Smart Cities, the incorporation of information technology into each and every part of urban life is happening. For the evolution of scalable, resourceful and dependable IoT centered Smart City, numerous technical and industrial challenges have risen owing to the volatile progress of IoT and Smart City applications. Some of the Smart City application comprises of smart mobility, smart services, smart utilities, smart economy, smart buildings, smart environment, smart governance, smart healthcare, smart citizens etc. [3].

Localization of a device is one of the central difficulties in mobile computing. Due to the necessity and significance of geo-location information, the design and employment of geo-location systems became plausible. The development of internal geo-location systems is the outcome of imprecision and failure of GPS (Global Positioning System) for internal and municipal locations [4].

The position information obtained is affected by the location and mobility of mobile nodes. With static nodes, the position information obtained through these systems is more exact. Due to the inevitable errors happening in distance specimens of a mobile node, tracing a constantly moving device is problematic. However, if the locations of these nodes stay steady all through averaging procedure these errors can be filtered out [5]. Another critical feature of geolocation awareness is the particular route of a mobile device. The alteration ability of a user interface to a user route will be exceedingly favorable for the system. Development, scalability, management, privacy, performance, and administration are the features of a geo-location system controlled through its architecture [6].

The Indoor Positioning System (IPS) tracks and locates a device within an indoor environment. With the launch of the Internet of Things (IoT), there has been a widespread rise in commercial and investigative interests of location-aware systems. Hence, a remarkable precision of position information is desired. Indoor Positioning System scholars are occupied for improvement of several localization methods to this time [7].

Location-based routing or Geocast routing uses the positioning information for routing. Some of the protocols that use GPS for localization are GAMER, Voronoi, GeoGRID, LBM, UARAD and TORA [8]. Some locationaware system that does not use GPS as an information source includes Cricket, Active Bat, Active Badge, Dolphin, RADAR, Ubisense and FIND. These systems can be used for internal positioning.

\section{PAPER ORGANIZATION}

The section wise paper organization is illustrated as follows. Section 3 covers the overview of different indoor locationbased systems in IoT with location accuracy less than $1 \mathrm{~m}$. Section 4 details the nomenclature and definition of the metrics to be considered for evaluation of the protocols. Section 5 contains the discussion on the protocols based on the metrics mentioned in the previous section. Section 6 concludes the paper precisely.

\section{SYSTEM OVERVIEW}

The overview of indoor location systems is described in this section. Their main properties, working techniques, advantages, and disadvantages have been discussed. 


\subsection{Cricket}

Cricket is an indoor location system developed as an anticipation of Project Oxygen by MIT. It uses an ultrasonic signal mechanism. Environmental requirements include sensor-based and pervasive computing. Applications running on sensor nodes, laptops and other mobile devices acquire fine position co-ordinates, narrow grid-based location information, and orientation from it.

Cricket has two main versions. In comparison, the accuracy and energy efficiency of Cricket version 2 is considerably more than Cricket Version 1. Cricket version 2 has improved continued object tracking and auto configuration algorithms.

Cricket is expected to work efficiently in urban areas and interiorly where GPS normally fails. A large collection of sensors can be combined with Cricket due to its low power operation and usage as location-aware sensor computing node [9].

Cricket has two vital advantages following its establishment on active beacons and passive listeners. This implies that it has no central beacon or location database which results in enhanced scalability and comfortable deployment.

Cricket possesses a range of $5 \mathrm{~m}$ with an alignment precision of 3 degrees and point approximation precision of $10 \mathrm{~cm}$. The data rate of the system is approximated at $1 \mathrm{~Hz}[10]$.

\subsection{Active Badge}

Active Badge is an Infra-Red (IR) signaling based locationaware system. Active Badge system finds the position of an individual by looking for its Active Badge location. It uses proximity algorithm for detecting locations.

Every 10-15 seconds a rare IR signal is transmitted by Active Badge pinned to an individual. All these periodic transmissions are picked up by sensors deployed in each room of a host building. A master station processes the information received from these sensors, which is then used to figure out the position of the badge (associated to an individual) [11].

The signaling rate is a substantial design problem due to power utilization of active signaling. This problem is somehow handled by restricting the Badge to transmit every $10-15$ seconds. The batteries present in the badge will last for almost a year due to small mean current usage. To sustain battery life, Active Badge is integrated with a light dependent element which turns the badge off when it is dark. Badge signal time is also increased when the lighting time is reduced, resulting in reduced current usage.

A major drawback of Badge signaling after every 10-15 seconds is that the location is only available for a few second windows. Nevertheless, this drawback is taken care of by the fact that the movement of an individual indoors is comparatively slower than outdoors. Therefore, the location provided by the Active Badge is quite precise at room size [12].

The particulars of the Active Badge system consist of $7 \mathrm{~cm}$ accuracy in a $5 \mathrm{~m}$ range. Its transmission data rate is $0.1 \mathrm{~Hz}$. Additional hardware is required for its deployed which renders its deployment cost slightly higher than other systems. Sensitivity to light is one of its limitations.

\subsection{Active Bat}

Active Bat, an ultrasonic indoor location system that trails small scale tags also known as bats. It is sketched on the perception of echolocation of Bats. Sonar echoes rebounding from an obstacle in the form of frequency are used for obstacle discovery and prevention.

Distance measurement on the basis of time is used as a basic mechanism for location discovery. The distance is measured through time taken from transmission to reception. The position of an object can be estimated by discovering the relative location of two or more Bats coupled to it. Active Bat is centralized as opposed to Cricket.

Its leading advantages include wireless connectivity, extreme precision, low-power, and cost-effectiveness. However, its drawbacks are complexity, average battery life, and redundant beacon demands. Each bat has a battery life of about 15 months [13]. The major specifications of Active Bat comprise of $50 \mathrm{~m}$ range with an accuracy of $3 \mathrm{~cm}$. $75 \mathrm{~Hz}$ data rate has been approximated for Active Bat systems.

Image processing, scheduling, feature selection, and classification are a few of the sectors where Active Bat has been employed.

\subsection{Dolphin}

DOLPHIN is short for Distributed Object Locating System for Physical-space Internetworking [14]. It is characterized as an ultrasonic indoor location-aware system. The system adopts the identical positioning approach as that of Cricket and Active Bat systems.

Scattered wireless sensor nodes are a main part of the DOLPHIN system. The hop-by-hop positioning technique is the key concept applied in DOLPHIN system. Both the RF and Ultrasonic signals are transmitted and received by the system. Distinct indoor objects have the nodes responsible for communication connection to them. The hop-by-hop positioning technique is the key concept applied in DOLPHIN system.

The main purpose behind the design of DOLPHIN system is the need to avoid manual configurations. To locate all the nodes inside the system, the system needs merely a limited number of already configured nodes as opposed to Cricket and Active Bat systems. Location detection of the objects in DOLPHIN system is obtained in a distributed fashion, which is commonly accomplished through the use of a few manually configured nodes. The system helps locate the object positions with least possible configurations. This is achieved through the use of distributed location algorithms.

DOLPHIN system has two main interests i.e. Firstly, as mentioned above, to locate all the nodes in a system it needs only a few pre-configured nodes. Secondly, even if there is no ultrasound reception from configured nodes, nodes can easily figure out their location. Its main drawback is its cost requirement.

In a room with $3 \mathrm{~m} \times 3 \mathrm{~m}$ dimensions, an accuracy of $2 \mathrm{~cm}$ can be achieved. Its installation has medium costs and uses a data rate of $20 \mathrm{~Hz}$ [10].

\subsection{Ubisense}

Ubisense is an ultra-wideband (UWB) [15] based real-time indoor positioning system developed by Ubisense Company [16]. The major components contained in Ubisense are the small tags connected to objects being located and the 
sensors spread over the targeted area. For communication purposes with the network, these tags are equipped with UWB transmitters and radio receivers. It contains all the hardware, firmware, and server-based versions of components.

UWB technology assists in reducing multipath and accuracy issues. This technology uses shortly pulsed (less than 1ns) electromagnetic waves transmitted in a wide frequency range i.e. greater than $500 \mathrm{MHz}$. These electromagnetic waves aid in filtering the reflected and time scaled copies of signals from the original signals. Signals transmission in UWB happens over a range of frequencies $(3.1-10.6 \mathrm{GHz})$ at the same time as opposed to RF technologies (transmission happens over a single frequency at one time). Target position in UWB signal is determined by the use of TDOA (Time Difference of Arrival and AOA (Angle of Arrival). For 3D positioning, two sensors are needed to be aware of the tag location.

Its principal advantages include multipath correction and accuracy. Low power consumption is another aspect of this technology that broadens the range of commercial applications. Its main drawbacks are the complex installation process, cost - ineffectiveness and medium privacy as the system is always aware of the location of a person.

Its prime specifications include an accuracy of $15 \mathrm{~cm}$ over a $15 \mathrm{~m}$ range with $1 \mathrm{~Hz}$ data rate for communication.

\subsection{Find}

The term FIND stands for Framework for Internal Navigation and Discovery. It is a simplified way to achieve indoor positioning. It lets us gather indoor location information through the use of our computer (Wi-Fi enabled) or smartphones. Location can easily be discovered inside the homes (bedroom, kitchen, living room etc.) or offices.

FIND was designed with an aspiration to replace motion sensors. Therefore, because of its high resolution, it can replace any motion sensors deployed. It can supply explicit location and user-particular information.

The system can be structured by using Raspberry Pis. They are arranged around the place in order to receive various signal strengths from different Wi-Fi devices. These Raspberry Pis circulates inquiry requests to all the Wi-Fi devices in its proximity and forwards this data to FIND server. It is called fingerprint data. The FIND server assembles this fingerprint data and forwards it to the main server. These requests occur every few minutes through a scan of their proximities. The man server then processes the fingerprint data and provides a precise location [17].

FIND can be used as an information source for operations performed through the data collected by motion sensors and GPS. However, apart from this, it provides indoor positioning making the overall system more accurate than the systems mentioned. The geolocation accuracy of FIND is below 10 sq. $\mathrm{ft}$. which puts it way ahead of GPS.

One of its leading advantages of FIND system is that its open source and easily configurable. These characteristics make it easy to use and deploy. Another main advantage of FIND is its almost no battery usage. It uses already existing applications and battery usage is next to none.
Another version of FIND available is FIND3. It is an application and does not need any hardware implementation. It has built-in passive scanning and supports data reception for any source [18]

The location information obtained through FIND may be used of in an extensive variety of applications from smallscale lifestyle tracking and home automation to large-scale business applications.

\section{METRICS OF INTEREST}

This section gives us an insight into the parameters considered for system comparison. The indoor location systems mentioned above are evaluated and compared on the basis of their scalability, range, accuracy, data storage, and privacy.

\subsection{Scalability}

Scalability is a feature of a network or a system, that depicts its capacity to grow, improve and control increasing requirements. A scalable system or network is known to be more complaint to the changing and increasing demands of its consumers.

Scalability is generally an indication of stability and ambition. A scalable network or system shows the tendency to manage the flow of elevated demands, production, trends, and challenges. In terms of location awareness, the scalability signifies the inclination towards evolution, indoor pervasiveness, and deployment.

\subsection{Range}

Range is defined as the period between two data points on a specific scale. It is the space containing the maximum and minimum values possible of a network or a system. Any set of values between the maxima and minima can be observed for a system. The range of a system refers to the degree of flexibility of a system.

In terms of location awareness, the range describes the scale of distance in which the system performance is favorable.

\subsection{Accuracy}

Accuracy is termed as the measure of closeness of a calculated amount to a standard amount in a system. Moreover, accuracy is also regarded as the inaccuracy ratio of determined value to a probable array of values.

Distance is used as the basis of accuracy measurement in case of geo-location systems. The variance of the location reported by a system and its real location amounts to accuracy.

\subsection{Data Storage}

Data Storage refers to the cumulative processes of saving data on a storing device. Data retention by a computing device for processing depends upon the process of storage. Storage is a crucial factor in sustaining information obtained from variable sources.

In the case of location-aware systems, storage is the technique through which the data observed by sensors is collected and processed. The location of the object is found on the basis of this data.

\subsection{Privacy}

Privacy also labeled as data privacy or information privacy is the ability of a system to preserve personal data securely without the access of third parties. A number of factors, 
methods, and mechanics involved in the process of securing delicate personal data comes under the concept of privacy.

In location-based systems, the privacy is the qualification of the system to hide the sensitive location information of users from third parties. The statistics contain the location as well as at what time and for how long the location was visited. Privacy concerns are a major hurdle in the rapid successful deployment of Smart City IoT applications.

Table 1: Properties of Indoor Location Systems

\begin{tabular}{|l|l|l|l|l|}
\hline System & Scalability & Accuracy & Storage & Privacy \\
\hline Cricket & High & $1-3 \mathrm{~cm}$ & Local & High \\
\hline Active Bat & Low & $3 \mathrm{~cm}$ & Central & High \\
\hline Active Badge & Low & $7 \mathrm{~cm}$ & Central & High \\
\hline Dolphin & Low & $2 \mathrm{~cm}$ & Central & Medium \\
\hline Ubisense & Low & $15 \mathrm{~cm}$ & Central & Medium \\
\hline FIND & High & $10 \mathrm{sq} . \mathrm{ft}$. & Central & High \\
\hline
\end{tabular}

\section{DISCUSSION}

The comparison of indoor location systems is presented in Table 1, as shown above. Considering the metrics mentioned the discussion starts with the Scalability of each system.

Comparing the scalability of the systems it is seen that two systems i.e. Cricket and FIND show high scalability while all others have low scalability. Therefore, Active Badge, Active Bat, DOLPHIN, and Ubisense are used as indoor location systems when fixed and specific tasks are being performed. But whenever a system is needed for wide range of applications and demands Cricket and FIND are a better match.

The second metric considered was the overall range of a system. The range is the area surrounded by a beacon in which signals can be transmitted and received. All the systems described have different ranges for a single beacon. The FIND system has the highest range with Active Bat, Ubisense, Cricket, Active Badge and Dolphin closely following respectively. However, the range becomes irrelevant if the accuracy of the systems is compromised.

For geo-location systems, accuracy is well-thought-out as one of the ultimate imperative parameters. According to Table 1, the most accurate system is DOLPHIN but due to its small range, it is not preferable. The Cricket system follows it closely with $1-3 \mathrm{~cm}$ accuracy within a $5 \mathrm{~m}$ range. Among all the systems Cricket is most favorable when it comes to accuracy of a system. FIND has an accuracy of 10 sq. ft. with the highest range which makes it even a better choice than Cricket.

Data storage is one of the main parameters as it contributes to the deployment cost of a system. Cricket is the only system which stores and processes the data locally i.e. it doesn't need any central control which makes it the perfect choice when it comes to cost-effectiveness. All the other systems need a central infrastructure to store and process data.
The privacy of a system is the one parameter where no compromise can be made. Privacy breach in a smart city would cause substantial damage to an individual. It not only compromises the home and office of an individual but also helps in deducing his routine, fitness, practices, lifestyle etc. The social life of an individual can similarly be assumed from co-location information. All the locationaware systems described have shown higher privacies except Dolphin and Ubisense which depict medium-high privacy.

\section{CONCLUSION}

In this paper, a comparison between various indoor location system for use in a smart city with high accuracy and small size has been administered. They are evaluated on the basis of their stability, range, accuracy, infrastructure requirements and privacy.

Each system has its own efficiencies and deficiencies. After the discussions in the above section, it is clear that two systems appear victorious i.e. Cricket and FIND. Their ease of use, low cost, privacy concerns and promising characteristics put them ahead of all others.

However, Cricket requires a sensor node or device always connected or carried by a person for location awareness but FIND works effortlessly with our smart devices. There is no need to carry or pin extra devices for indoor geocasting purposes.

A concept of BYOD (bring your own device) is emerging which allows the people to carry or bring their own devices everywhere. For FIND system this concept can be easily used for positioning without carrying an extra beacon. The emerging fame of smart devices among users makes it even easier to deploy.

Additionally, no battery usage of FIND also makes it the most promising system for indoor positioning in smart cities. 


\section{REFERENCES}

[1] Čabarkapa, Danijel, Ivana Grujić, and Petar Pavlović., 2015. "Comparative analysis of the bluetooth lowenergy indoor positioning systems." In Telecommunication in Modern Satellite, Cable and Broadcasting Services (TELSIKS), 2015 12th International Conference on, pp. 76-79. IEEE, 2015.

[2] Smart City, https://www.techopedia.com/definition/ 31494/smart-city

[3] Eckhoff, David, and Isabel Wagner., 2017. "Privacy in the Smart City-Applications, Technologies, Challenges, and Solutions." IEEE Communications Surveys \& Tutorials 20, no. 1 (2017): 489-516.

[4] Rida, Mohamed Er, Fuqiang Liu, Yassine Jadi, Amgad Ali Abdullah Algawhari, and Ahmed Askourih., 2015. "Indoor location position based on bluetooth signal strength." In Information Science and Control Engineering (ICISCE), 2015 2nd International Conference on, pp. 769-773. IEEE, 2015.

[5] Halder, Subir, and Amrita Ghosal., 2016. "A survey on mobile anchor assisted localization techniques in wireless sensor networks." Wireless Networks 22, no. 7 (2016): 2317-2336

[6] Priyantha, Nissanka Bodhi, Hari Balakrishnan, Erik D. Demaine, and Seth Teller., 2005. "Mobile-assisted localization in wireless sensor networks." In INFOCOM 2005. 24th Annual Joint Conference of the IEEE Computer and Communications Societies. Proceedings IEEE, vol. 1, pp. 172-183. IEEE, 2005.

[7] Rusli, Mohd Ezanee, Mohammad Ali, Norziana Jamil, and Marina Md Din., 2016. "An improved indoor positioning algorithm based on rssitrilateration technique for internet of things (iot)." In Computer and Communication Engineering (ICCCE), 2016 International Conference on, pp. 7277. IEEE, 2016.
[8] Prasanth, K., and P. Sivakumar., 2014. "Location based routing protocol-A survey." In Computer Communication and Informatics (ICCCI), 2014 International Conference on, pp. 1-6. IEEE, 2014.

[9] Cricket, http://cricket.csail.mit.edu/

[10] Koyuncu, Hakan, and Shuang Hua Yang., 2010. "A survey of indoor positioning and object locating systems." IJCSNS International Journal of Computer Science and Network Security 10, no. 5 (2010): 121128.

[11] Active Badge, http://www.cl.cam.ac.uk/research/dtg/ attarchive/ab

[12] Kumareson, P., R. Rajasekar, and P. Prakasam., 2015. "Accurate Location Identification Using Bluetooth in Android Smart phone." Int. Dly. J 30 (2015): 81-86.

[13] Batistić, Luka, and Mladen Tomic., 2018. "Overview of indoor positioning system technologies." In 2018 41st International Convention on Information and Communication Technology, Electronics and Microelectronics (MIPRO). IEEE, 2018.

[14] Minami, M.; Fukuju, Y.; Hirasawa, K.; Yokoyama S.; Mizumachi, M.; Morikawa, H.; Aoyama, T.; 2004. Dolphin: A practical approach for implementing a fully distributed indoor ultrasonic positioning system, Ubicomp, 347-365.

[15] Alarifi, Abdulrahman, AbdulMalik Al-Salman, Mansour Alsaleh, Ahmad Alnafessah, Suheer AlHadhrami, Mai A. Al-Ammar, and Hend S. AlKhalifa., 2016. "Ultra wideband indoor positioning technologies: Analysis and recent advances." Sensors 16, no. 5 (2016): 707.

[16] Ubisense Company, https://www.ubisense.net/

[17] FIND, https://github.com/schollz/find-lf

[18] FIND3, https://www.internalpositioning.com/doc/faq .md 